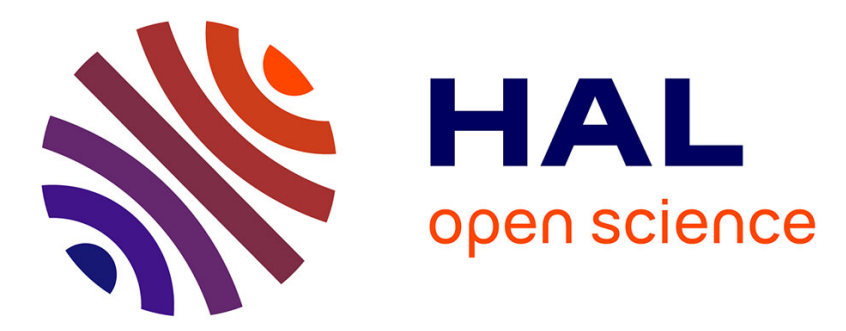

\title{
From yield to fracture, failure initiation captured by molecular simulation
}

\author{
Laurent Brochard, Ignacio G. Tejada, Karam Sab
}

\section{To cite this version:}

Laurent Brochard, Ignacio G. Tejada, Karam Sab. From yield to fracture, failure initiation captured by molecular simulation. Journal of the Mechanics and Physics of Solids, 2016, 95, pp.632 - 646 . 10.1016/j.jmps.2016.05.005 . hal-01686201

\section{HAL Id: hal-01686201 https://hal.science/hal-01686201}

Submitted on 20 Jun 2018

HAL is a multi-disciplinary open access archive for the deposit and dissemination of scientific research documents, whether they are published or not. The documents may come from teaching and research institutions in France or abroad, or from public or private research centers.
L'archive ouverte pluridisciplinaire $\mathbf{H A L}$, est destinée au dépôt et à la diffusion de documents scientifiques de niveau recherche, publiés ou non, émanant des établissements d'enseignement et de recherche français ou étrangers, des laboratoires publics ou privés. 


\title{
From Yield to Fracture, Failure Initiation Captured by Molecular Simulation
}

\author{
Laurent Brochard ${ }^{\mathrm{a}, *}$, Ignacio G. Tejada ${ }^{\mathrm{b}}, \mathrm{Karam}_{\mathrm{Sab}}^{\mathrm{a}}$ \\ ${ }^{a}$ Université Paris-Est, Laboratoire Navier (UMR 8205), CNRS, ENPC, IFSTTAR \\ $6 \& 8$ avenue Blaise Pascal, 77455 Marne-la-Vallée, France. \\ ${ }^{b}$ Université Grenoble Alpes, 3SR, CNRS \\ Domaine Universitaire BP53, 38041 Grenoble Cedex 9, France
}

\begin{abstract}
While failure of cracked bodies with strong stress concentrations is described by an energy criterion (fracture mechanics), failure of flawless bodies with uniform stresses is captured by a criterion on stress (yielding). In-between those two cases, the problem of failure initiation from flaws that moderately concentrate stresses is debated. In this paper, we propose an investigation of the process of failure initiation at the atomic scale by mean of molecular simulations. We first discuss the appropriate scaling conditions to capture initiation, since system sizes that can be simulated by molecular mechanics are strongly limited. Then, we perform a series of molecular simulations of failure of a 2D model material, which exhibits strength and toughness properties that are suitable to capture initiation with systems of reasonable sizes. Transition from fracture failure to yield failure is well characterized. Interestingly, in some specific cases, failure exceeds yield failure which is in contradiction with most initiation theories. This occurs when stress are highly concentrated while little mechanical energy is stored in the material. This observation calls for a theory of initiation which requires that both stress and energy are necessary conditions of failure. Such an approach was proposed by Leguillon (2002). We show that the predictions of this theory are consistent with the molecular simulation results.
\end{abstract}

Keywords: Failure initiation, molecular simulation, fracture, yield

\footnotetext{
*Corresponding author

Email address: laurent.brochard@enpc.fr (Laurent Brochard)
} 


\section{Introduction}

Understanding the mechanical failure of materials is of prime concern for the security and reliability of man-made structures. Yet, the initiation of failure under moderate stress concentrations is still very debated and there is no clear consensus in the scientific community about a generic failure criterion that could predict initiation. In this paper, we show that failure initiation behavior can be evidenced at the atomic scale with classical molecular mechanics simulations, considering the case of a 2D model material. This work opens the perspective to revisit the fundamentals of failure initiation starting from where failure originates, that is atoms and atomic interactions.

Mechanical failure of a material with a pre-existing crack is captured by the theory of Linear Elastic Fracture Mechanics (LEFM) initially formulated in the pioneering works of Griffith (1921) and Irwin (1957). According to LEFM (Anderson, 2005), the pre-existing crack propagates if the mechanical energy $G$ that is released upon the crack advance exceeds a critical value $G_{c}$, a.k.a. critical energy release rate: $G \geq G_{c}$. The stress and strain fields are singular at the crack tip with a term in $1 / \sqrt{(} r)$ in their asymptotic development (where $r$ stands for the distance to the crack tip). The energy released upon the crack advance is related to this singularity according to Irwin's formula, e.g., for a mode I loading in plane stress: $G=K_{I}^{2} / E$, where $E$ is the Young's modulus and $K_{I}=\lim _{x \rightarrow 0^{+}} \sqrt{2 \pi r} \sigma_{y y}(y=0)$ for a crack orthogonal to the $y$ direction. The critical stress intensity $K_{I c}=\sqrt{E G_{c}}$ is called the toughness. Even if the existence of a singularity at the crack tip is questionable, LEFM has been successful at predicting the failure of precracked structures under the small scale yielding assumption, i.e., the region of yielding at the crack tip is much smaller than the size of the structure. In the following of the paper, we will refer to this type of failure as 'fracture failure'.

In contrast, in the absence of stress concentration, the failure of materials is caused by an excessive stress or strain instead of energy released. The collection of critical stresses or strains constitutes a failure surface, and the corresponding failure criterion takes the form $f(\sigma$ or $\varepsilon) \geq 0$. In the following of the paper, we will refer to this type of failure as 'yield failure'. Accordingly, the criterion describing the failure of an intact material differs radically from that describing the failure of a pre-cracked material, down to the very nature of the apparent cause of failure: stress or energy.

These two cases, a pre-cracked body and an intact material, are very 
particular cases of structures. In reality, structures are most likely to contain flaws with moderate stress concentrations such as notches or holes. These stress concentrations are 'moderate' because the singularities are in $1 / r^{\alpha}$ with $\alpha<1 / 2$ (for notches) or because the stress field is not singular at all (for holes, blunted notches etc.). Accordingly, the stress intensity factor and energy release rate are zero for this type of flaws, $K_{I}=G=0$, and thus the energy criterion of LEFM is never verified: the structure should never fail regardless of the loading. In contrast, because of the singularity at a notch tip, the yield surface criterion is always verified: the structure should always fail regardless of the loading. This simple observation demonstrates that failure originating from 'moderate' stress concentrators cannot be predicted by any of the two criteria (stress or energy) and a more universal criterion is needed.

\section{Theories of initiation}

Many theories and criteria have been proposed to predict failure initiation. One of the first approaches that reconciled fracture failure and yield failure were the Cohesive Zone Models (CZM) originally proposed by Dugdale (1960) and Barenblatt (1962). CZM consist in introducing cohesive forces between the crack faces which oppose the opening of the crack and suppress the non-physical singularity at crack tips. CZM approaches, which capture the yielding inside the cohesive zone, are equivalent to LEFM under small scale yielding, but predict yield failure when the size of the cohesive zone becomes significant (Anderson, 2005). In addition to the yield stress and critical energy release rate, CZM introduce an additional material property, the cohesive law, which relates the cohesive force to the crack opening. The cohesive law is not a totally independent property since its integration must be equal to the critical energy release rate. Only the shape of the law is a new property which can significantly affect failure initiation. Various shapes have been considered in the literature depending on the nature of the material such as the 'plastic' (constant) law for metals (Dugdale, 1960). Application of CZM requires to specify a priori the path of the initiated crack which is known unambiguously for the modeling of interface debonding (Marigo and Truskinovsky, 2004) or for the simple loading and geometries (Ferdjani et al., 2007), but which is a limitation in the general case.

While CZM localize the yielding in a strip at the crack tip, it is not suited for a numerical implementation in Finite Element Methods (FEM) in which 
elements of finite volumes are considered. Hillerborg et al. (1976) proposed a non linear FEM approach to capture crack initiation in concrete structures. This approach produces bands of yielding where cracking initiates that are analogous to cohesive zones, but over elements of finite volume. The associated cohesive law is decreasing to capture the micro-cracking prior to (macro-)cracking. Bažant and Cedolin (1979) followed with the crack band theory built on the same principle. These FEM approaches proved objective (Cedolin and Bažant, 1980; Bažant and Cedolin, 1980, 1983) and ensure continuity between fracture failure and yield failure (Bažant, 1984). Similarly, continuum damage mechanics approaches which consider a damage variable to captures the dissipative processes in a finite volume (e.g., microcracking) can be used to predict initiation with a criterion on the damage variable (Chaboche, 1981; Lemaitre, 1986). An advantage of these FEM approaches over CZM is that one does not need to specify a priori the path of the initiated crack. However, one has to introduce a characteristic length (or volume), the physical meaning of which is not straightforward (e.g., a few aggregate sizes are suggested for concrete and rocks (Bažant, 1984)).

Alternatively, the non-local approach has been proposed by Novozhilov (1969) to capture initiation: failure occurs when the average ('non-local') stress over a small distance from the point of singularity equals the yield stress. Owing to the integrability of the $1 / \sqrt{(} r)$ singularity in the case of a pre-cracked body, this criterion leads to a finite non-zero fracture failure consistent with LEFM; while predicting yield failure for an intact body. A new parameter, the length of averaging, is introduced but is necessarily related to the yield stress and toughness to ensure consistency with LEFM. Non-local approaches are applicable to any 'moderate' stress concentrations and loading modes and can be used to predict the crack orientation (Seweryn, 1994; Seweryn and Mróz, 1995; Seweryn, 1998). A criterion of similar formulation is that proposed by Ritchie et al. (1973): failure occurs if the stress exceeds the yield stress over a characteristic length. Interestingly, the criterion of Ritchie et al. (1973) was proposed in the context of plastic materials, whereas Novozhilov (1969) consider elastic brittle materials down to the atomic scale. And yet both formulations are very similar.

Finally, Finite Fracture Mechanics (FFM) approaches were proposed to capture crack initiation (Hashin, 1996): whereas LEFM considers an infinitesimal crack advance, FFM considers crack advances of finite length (initiated cracks appear with finite length). While the LEFM failure criterion is never satisfied for moderate stress concentrations (notches and holes), the 
energy released by a finite crack advance does reach the critical value at finite loading. Although FFM general principle is analogous to LEFM, in practice it takes the form of a complex minimization problem over the set of possible crack paths. Francfort and Marigo (1998) proposed a variational formulation to solve this minimization problem. In the case of a pre-cracked body FFM and LEFM are equivalent (limit of infinitesimal crack advance), but FFM ability to predict failure in a wider range of stress concentration makes it more versatile and physically relevant (Marigo, 2010). In itself, FFM does not reduce to a criterion on stress in the absence of stress concentration. To capture yield failure, the variational approach of Francfort and Marigo (1998) has been combined with cohesive zone interfaces instead of a Griffith (infinitely thin) surface (Laverne and Marigo, 2004; Charlotte et al., 2006; Bourdin et al., 2008), thus offering a more general application of CZM since the path of initiated crack is no more needed a priori. Another approach has been proposed by Leguillon (2002) to capture yield failure in FFM, the application of which is simpler than cohezive zone interfaces since it relies on a linear elastic calculation only: failure occurs if the energy criterion of FFM is satisfied and if the yield failure criterion is verified along the path of the initiated finite crack. This criterion has been applied to failure from notch and holes (Leguillon et al., 2007; Leguillon and Piat, 2008; Martin et al., 2012) and is supported by some experiments (Romani et al., 2015). A initiation criterion very close to that proposed by Leguillon (2002) has been considered by Li et al. (1995) earlier to study desiccation. In this formulation, the yield failure has to be attained only at the point of initiation. The former criterion (Leguillon, 2002) seems more general than the later (Li et al., 1995) which does not seem applicable to notched bodies (one cannot evaluate yield only at the tip because of the stress singularity). A particular feature of FFM approaches is that the initiated crack length is not a material property but depends on the geometry of the structure, which is contradictory with theories considering a fixed characteristic length (non-local approaches, FEM with crack band or damage). Whether or not one should consider a characteristic length of the material as a third material property (with strength and toughness) to describe failure initiation is a debated question. Material properties other than a characteristic length have also been considered as third material parameter (Li and Zhang, 2006).

Apart from stress concentration, transition from fracture failure to yield failure is also a matter of scale. A material is flaw tolerant (or flaw insensitive) when the characteristic dimension of its structure is comparable to 
the characteristic length $l_{0}=\left(K_{I c} / \sigma_{\text {yield }}\right)^{2}$ Gao and Chen (2005), where $K_{I c}$ is the toughness and $\sigma_{\text {yield }}$ is the yield stress. $l_{0}$ quantifies the process zone size at a crack tip. At such a scale, the small scale yielding hypothesis does not apply and LEFM would predict failure at a stress exceeding yield failure. Instead, one expects yield failure to prevail over fracture failure. Flaw tolerance is thus defined as the capacity of a material to be insensitive to fracture failure. Flaw tolerance is commonly observed in biological materials such as bone, spider silk or nacre Gao et al. (2003); Gao (2006); Ritchie et al. (2009), but also in polycrystalline materials Gu et al. (2013); Zhang et al. (2012). A seemingly accepted principle of flaw tolerance is that yield failure is an upper bound to failure, which is consistent with some of the initiation theories (e.g., CZM).

In the view of this short, certainly non-exhaustive, review of existing theories of initiation, it is clear that no consensus exists in the scientific community. The inherent differences between materials (microstructure, mechanical behavior) may well be the primary reason why no unified theory as emerged. But, an other important reason is that the physics of failure initiation is still poorly understood. As an attempt to improve our understanding of initiation, we propose here to investigate failure initiation by molecular simulation. Since failure of brittle materials originates from the breaking of atomic bonds, the molecular scale seems the appropriate scale to develop a fine understanding of the physics of initiation. A lot of effort has already been dedicated to the atomistic study of fracture failure and yield failure, respectively. Yet, few molecular simulation works have focused on the question of initiation. Inoue et al. (1995), Cao and Wei (2007) and Pan and Rupert (2014) investigated crack initiation at grain boundaries (polycrystals). Fyta et al. (2006) investigated crack initiation in a nanocomposite containing strong inclusions in a soft matrix (crystals in polymer). Kumar et al. (2011) and Gu et al. (2013) investigated initiation from blunted notches in polycrystals. In all these works, failure initiation is strongly influenced by microstructure. In order to evaluate the fundamentals of initiation theories, one needs to address the question of initiation in a simple homogeneous system without interfaces. Interesting works in this respect are that of Lu et al. (2008), Zhang et al. (2012) and Brochard et al. (2015) who considered failure of initially flawed homogeneous materials. They showed that fracture failure can be observed for brittle solids (diamond, graphene and silica, respectively) even for very small cracks (a few atoms large), while only yield failure is observed on the same scale for more ductile materials (amorphous carbons). Most interest- 
ing with respect to initiation are the works that investigate the continuous transition from fracture failure to yield failure. Mattoni et al. (2005) and Zhang et al. (2012) studied the failure of pre-cracked silicon carbide and nano-crystalline graphene and observed deviations from fracture failure at small crack lengths $\left(\sim l_{0}\right)$. These deviations correspond to the flaw tolerant regime and Mattoni et al. (2005) showed that it could be captured with a CZM model (Bilby et al., 1963). Yield strength appears as an upper boundary to failure in this regime. Similarly, deviation from fracture failure at small crack lengths is observed by Yin et al. (2015) for pristine graphene, although the effect is more subtle because of the high strength of the material. Alternatively, Zhang et al. (2012) considered failure from elliptic flaws with major axis orthogonal to the loading direction, and varied the aspect ratio thus modulating the stress concentration. They showed that for nanocrystalline graphene (in the flaw tolerant regime), failure is insensitive to the aspect ratio, whereas for single-crystalline graphene (subject to fracture failure), failure stress increases continuously with stress concentration. The former case corresponds to yield failure only, and the later case is a deviation from fracture toward yield. To summarize, previous works have studied initiation in the regime of small flaws $\left(\sim l_{0}\right)$ or from elliptic flaws with major axis orthogonal to loading. In all these cases, failure never exceeded yield failure, and CZM model seem to capture the transition from fracture failure to yield failure for initiation from small cracks. No such confrontation with modeling was performed for initiation from elliptic flaws. Moreover, other situations may be of interest that have not been investigated yet: for instance, initiation from notch, from elliptic flaws with major axis in the direction of loading, or from large cracks where the size of the remaining intact material if of order $l_{0}$. In this paper, we present a molecular simulation study that investigates initiation from cracks and elliptic flaws. Initiation is modulated by changing the aspect ratio of the elliptic flaws or by changing the size of the crack. We explore in particular situations that have not been investigated yet in the literature. We capture the transition from fracture failure to yield failure, that we confront to existing initiation theories.

\section{Capturing initiation}

To capture the transition from fracture failure to yield failure, a simple approach consists in considering failure from flaws inducing stress concentrations that range from strong concentrations ( crack-like) to weak concentra- 


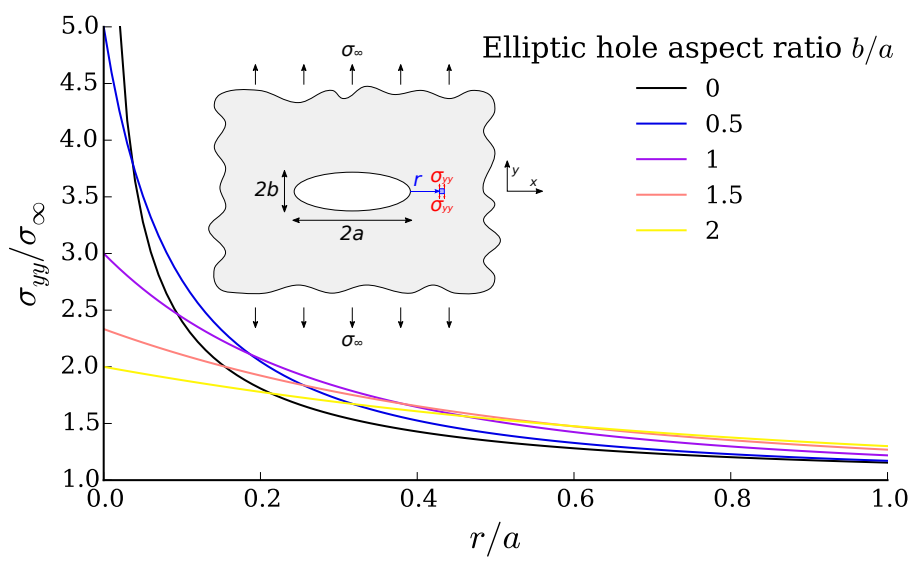

Figure 1: Stress concentration in the vicinity of an elliptic hole in an infinite body.

tions $(\sim$ uniform). This could be done with notches of various angles. Here, we consider elliptic holes of various aspect ratios instead. In the limit of infinitely thin elliptic holes, stress concentration approaches that of a crack. This was first noticed by Inglis (1913) considering the stress around an ellipse in an infinite body. We display in Figure 1 the stress profile in function of the aspect ratio for an elliptic hole in an infinite body (exact solution by (Maugis, 1992)). Although the stress near an elliptic hole is not singular, the crack-like singularity arises in the limit of a infinitely thin ellipse.

It is common in molecular simulations to consider periodic boundary conditions to mimic the state of a system in an infinite body. Doing so, one can avoid surface effects at non-periodic boundaries that are particularly strong at nanoscale and could affect the material behavior. In addition, when the typical length of inter-atomic interactions exceeds the system size, periodic boundary conditions are more suited to model the macroscopic material behavior (this is especially the case for electrostatic interactions). Accordingly, we consider the molecular simulation of failure of a periodic system with an initial elliptic hole in its center (see Figure 2). The system is loaded in the vertical direction in displacement, while the horizontal dimension is kept constant. The horizontal axis of the elliptic hole is set to half the system size, whereas we vary the vertical axis. The geometry is invariable in the transverse direction ( $\mathrm{z}$ ) so that we restrict ourselves to the $2 \mathrm{D}$ problem only. For a system periodic in the transverse direction, this set up corresponds to plane strain conditions. In this paper, we consider a $2 \mathrm{D}$ material with no 

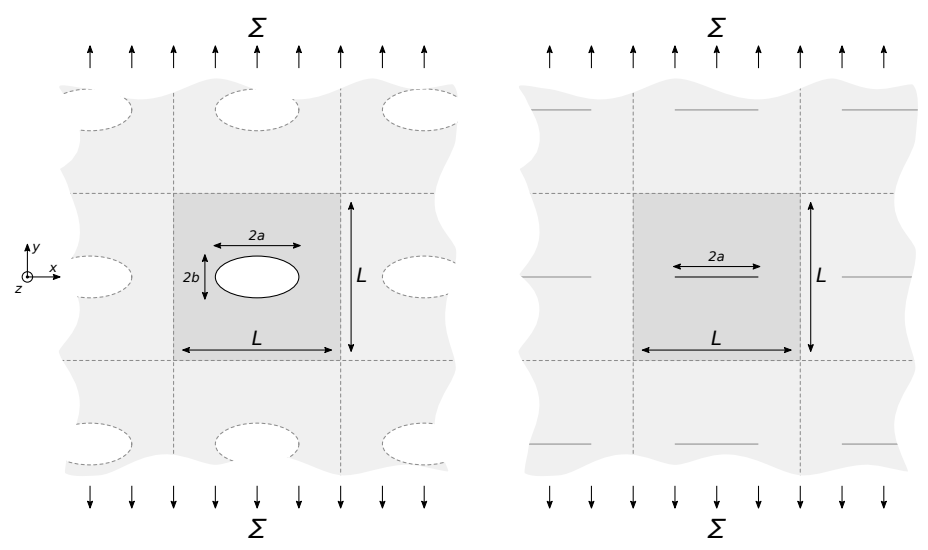

Figure 2: Periodic system considered in this work with elliptic flaws (left) and cracks (right).

third dimension, thus in plane stress conditions.

When the aspect ratio $b / a$ of the ellipse is small, fracture failure and crack-like stress singularity are expected. The stress intensity is that of a material with a doubly periodic array of cracks, which is of the form:

$$
K_{I}=\Sigma \sqrt{\pi a} C\left(\frac{2 a}{L}\right)
$$

where $\Sigma$ is the remote stress and $C(2 a / L)$ is a correction factor due to the presence of periodic replicas of a crack (in the limit $2 a / L \rightarrow 0, C \rightarrow 1$ and one recovers the usual stress intensity of a single crack in an infinite body). There exists no analytic expression of the correction factor $C(2 a / L)$, but several numerical approaches were proposed in the literature. We use here the numerical approach of Karihaloo et al. (1996), and we display in Figure 3 how the correction factor depends on the reduced periodic crack length $2 a / L$. As expected, it converges to 1 at small crack lengths and to $+\infty$ when the crack length reaches the periodic cell size $(2 a / L \rightarrow 1)$. The influence of the periodic replicas becomes significant for $2 a / L>0.5(C>1.11)$.

According to equation 1, the critical remote loading at failure is:

$$
\Sigma_{c r}=\frac{K_{I c}}{C\left(\frac{2 a}{L}\right) \sqrt{\pi a}}
$$

We adopt a dimensionless formulation of Equation 2 that is more convenient in our analysis: 


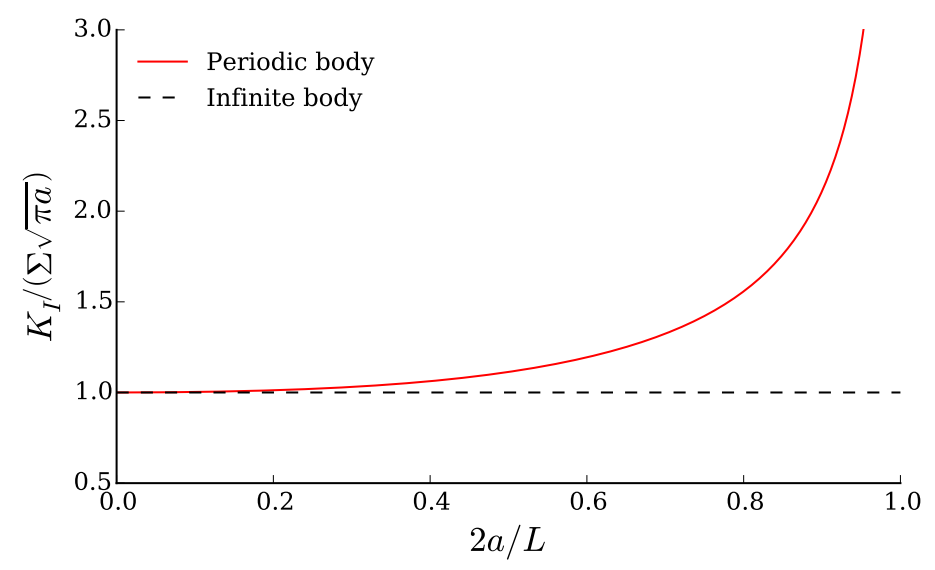

Figure 3: Stress intensity correction factor $C(2 a / L)=K_{I} /(\Sigma \sqrt{\pi a})$ for a body with a doubly periodic array of cracks in function of the reduced periodic crack length $2 a / L$. The limit case of an infinite body $K_{I} /(\Sigma \sqrt{\pi a})=1$ is displayed as a reference.

$$
\frac{\Sigma_{c r} \sqrt{L / 2}}{K_{I c}}=\left(C\left(\frac{2 a}{L}\right) \sqrt{\pi \frac{2 a}{L}}\right)^{-1}
$$

Accordingly, the dimensionless quantity $\Sigma_{c r} \sqrt{L / 2} / K_{I c}$ characterizing the critical stress of fracture failure is a function of the dimensionless crack length $2 a / L$. We display this relationship in Figure 4, along with the case of a single crack in an infinite body (the same dimensionless relationship applies without the correction factor $C(2 a / L))$. As expected, the critical stress diverges in $1 / \sqrt{a}$ at small crack lengths, and differ from the non periodic case at crack lengths approaching the periodic cell size. Irrespective of the system size $L$ and toughness $K_{I c}$, fracture failure of any material is expected to follow this master curve.

As for yield failure, it arises when the aspect ratio $b / a$ of the elliptic hole becomes large and the local curvature of the ellipse at the point of initiation tends to 0 . In the limit of yield failure, the stress is uniform, and the system is made of periodic vertical strips of thickness $L-2 a$. Yield failure occurs when the uniform stress in the strips reaches $\sigma_{\text {yield }}$, that is the remote stress on the overall material reaches:

$$
\Sigma_{c r}=\sigma_{\text {yield }} \frac{L-2 a}{L}
$$


Adopting the same dimensionless formalism as for fracture failure, we obtain:

$$
\frac{\Sigma_{c r} \sqrt{L / 2}}{K_{I c}}=\sqrt{\frac{L}{2 l_{0}}}\left(1-\frac{2 a}{L}\right)
$$

where $l_{0}=\left(K_{I c} / \sigma_{\text {yield }}\right)^{2}$ is a length characterizing the size of the yielding zone at a crack tip (Anderson, 2005). According to Equation 5, for yield failure, the dimensionless quantity $\Sigma_{c r} \sqrt{L / 2} / K_{I c}$ decreases linearly with $2 a / L$ from $\sqrt{L /\left(2 l_{0}\right)}$ for $2 a / L=0$ to 0 for $2 a / L=1$ (see Figure 4 ). Unlike for fracture failure, this curve is size dependent since the periodic cell size $L$ appears in the y-intercept $\sqrt{L /\left(2 l_{0}\right)}$. Two regimes are possible. For small periodic cell sizes with $L /\left(2 l_{0}\right)<1.96$, the critical stress for yield failure is always smaller than the critical stress for fracture failure. Hence the system is too small with respect to the crack process zone to observe any fracture failure, and only yield failure is expected. This 'yield-only' regime corresponds to a flaw tolerant material. It was observed by Lu et al. (2008) and Brochard et al. (2015) in the case of molecular simulation of amorphous carbons. For larger periodic cell sizes with $L /\left(2 l_{0}\right)>1.96$, the critical stress for yield failure is larger than the critical stress for fracture failure except when $2 a / L$ approaches 0 or 1 . In this regime, one should be able to capture fracture failure by considering the materials with initial cracks. Fracture failure was indeed observed by Lu et al. (2008) and Brochard et al. (2015) in the cases of diamond and silica, by Zhang et al. (2012) and Yin et al. (2015) in the case of single-crystalline graphene. Mattoni et al. (2005) and Zhang et al. (2012) also observed fracture failure for silicon carbide and nano-crystalline graphene, but noticed a deviation from fracture failure at small crack length $2 a / L$ which corresponds to the limit where yield failure no more exceeds fracture failure. Therefore, it seems possible to capture failure initiation provided that the system considered is large enough to satisfy the inequality $L /\left(2 l_{0}\right)>$ 1.96. Following this observation, in the present work, our investigation of the transition from fracture failure to yield failure is two-fold: 1- we study the failure behavior of pre-cracked body in order to observe deviation from fracture failure at low crack lengths when $2 a / L$ approaches 0 but also at large crack length when $2 a / L$ approaches $1 ; 2$ - we study the failure behavior in presence of elliptic holes of various aspect ratios $b / a$ for a given ellipse length $2 a / L$. 


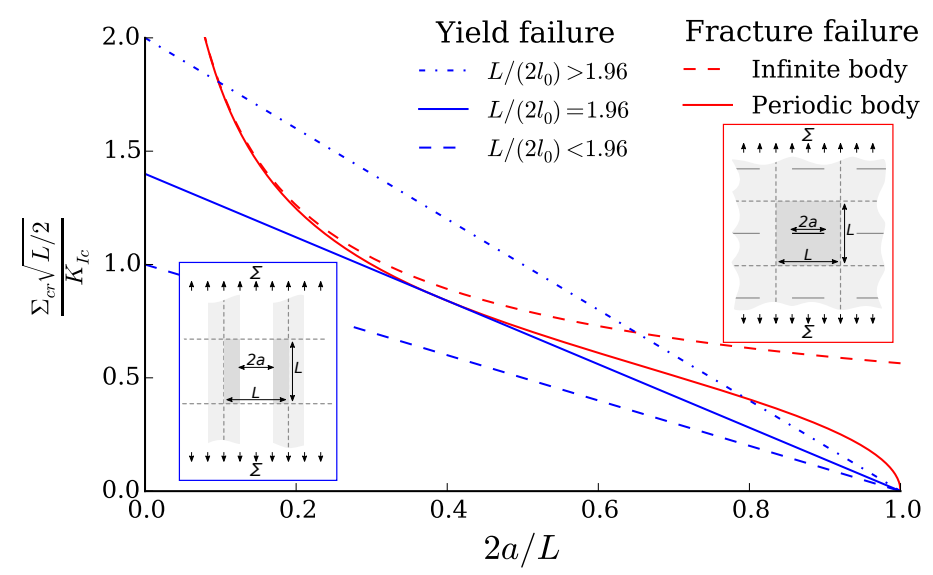

Figure 4: Fracture failure (red) and yield failure (blue) in function of the flaw size $2 a / L$. Two regimes are possible depending on the ratio $L /\left(2 l_{0}\right)=(L / 2) \cdot\left(\sigma_{\text {yield }} / K_{I c}\right)^{2}$. For $L /\left(2 l_{0}\right)<1.96$, yield failure is always lower than fracture failure, whereas for $L /\left(2 l_{0}\right)>$ 1.96, yield failure is higher than fracture failure except when $2 a / L$ approaches 0 or 1 .

\section{Molecular simulation of failure initiation in a $2 \mathrm{D}$ model material}

The case study we consider here is the failure of a two dimensional crystalline material inspired from graphene. Graphene is a material that has attracted a lot of attention recently because of its exceptional properties. However, these exceptional properties is not what motivates our choice here. Instead, we are interested in the simplicity of the material (a 2D lattice) which limits the computational cost, and, above all, the characteristic length $l_{0}=\left(K_{\text {Ic }} / \sigma_{\text {yield }}\right)^{2}$ we estimated is about $1.5 \mathrm{~nm}$, which is large enough for the process zone at crack tip to cover many atoms, but small enough to capture failure initiation by molecular simulation ( $L$ must well exceed $1.96 \cdot 2 \cdot l_{0} \approx 6$ $\mathrm{nm}$, which can be achieved easily with conventional molecular dynamics). In this respect, this $2 \mathrm{D}$ model material is an appropriate test case for the purpose of this study. Note that these simulations cannot be considered representative of real graphene. Indeed, we constrain the material in a 2D space, which is a great simplification in terms of computation, but is not the true behavior of graphene. Out of plane deformations are essential for the mechanics of graphene, in particular regarding failure (Moura and Marder, 2013; Dewapriya et al., 2014). Moreover, the interaction potential we use, based on the second generation REactive Bond Order (REBO) potential (Brenner et al., 2002), is known to significantly overestimate the strength 
and toughness of graphene as well as of nanotubes or diamond (Shenderova et al., 2000; Belytschko et al., 2002). Both the 2D constrain and the potential used enhance the resistance to failure. Assuming a thickness of $3.45 \AA$, the strength and toughness we obtained $(190 \mathrm{GPa}$ and $7.2 \mathrm{MPa} \cdot \sqrt{\mathrm{m}})$ are both about two times higher than previously reported values for graphene (100 GPa and $4 \mathrm{MPa} \cdot \sqrt{\mathrm{m}}$ (Zhao et al., 2009)). Therefore we do not pretend to simulate the true behavior of graphene. Instead, in the remaining of this article, we will refer to a '2D model material' to avoid confusion.

The 2D model material is a crystalline material made of atoms in the planar honeycomb lattice of graphene (see Figure 5). We performed molecular simulation of periodic cells of this material in which we introduced initial flaws (cracks and elliptic holes). We considered two sizes of periodic cells: $L=10 \mathrm{~nm}$ and $L=20 \mathrm{~nm}$. The lattice of graphene does not allow for perfectly square cells as displayed in Figure 2. Nevertheless, we consider cells that were almost square, with ratios $L_{y} / L_{x}$ of the cell dimension of 1.014 and 1.002 for the $10 \mathrm{~nm}$ and $20 \mathrm{~nm}$ systems, respectively. Non perfectly square cells is expected to have little effect on failure: for instance, in the case of fracture failure, the impact on the correction factor $C(2 a / L)$ is less than $0.1 \%$ (estimation from the numerical approach of Karihaloo et al. (1996)) We initiated cracks by forcing atoms across the line of the crack to ignore themselves as if they were not neighbors (see Figure 5). We varied the initial crack size from the size of a few atoms to almost the size of the cell, thus varying $2 a / L$ from 0 to 1 . We initiated holes by removing from the periodic cell all the atoms included in an ellipse (see Figure 5). The dimension of the initiated elliptic holes in the horizontal direction was always the same, fixed to half the periodic cell size $(2 a / L=0.5)$. We varied the dimension in the vertical direction from $b / a=0.1$ to $b / a=1.8$. These two types of initial flaws allow us to explore failure initiation in different manners as explained in the previous section. In all this work, we study failure of the $2 \mathrm{D}$ model material in the 'armchair' direction.

The molecular simulations we performed are classical Molecular Dynamics (MD) simulations, based on an empirical inter-atomic interaction potential. We used the second generation Reactive Bond Order Potential (REBO) (Brenner et al., 2002) coupled with a non-reactive harmonic approximation that improves the computational efficiency of the MD. The development of this coupling methodology and its application were the purpose of a previous work by Tejada et al. (2016). In short, a reduced non-reactive potential is built from the REBO potential as an harmonic approximation of it in the 

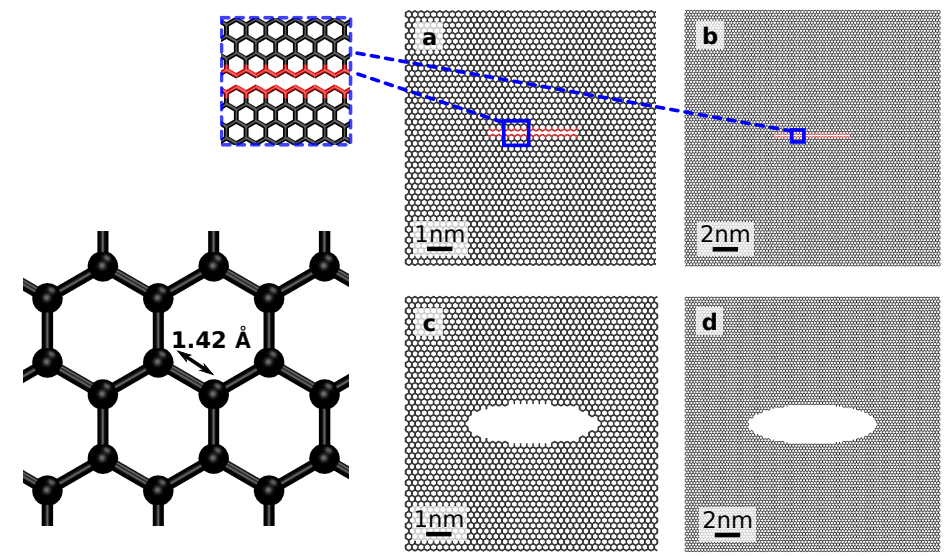

Figure 5: Hexagonal lattice of the 2D model material (left) and some of the system simulated (right). 'a' and 'b' are two pre-cracked systems for the $10 \mathrm{~nm}$ and $20 \mathrm{~nm}$ cells, respectively. 'c' and 'd' are two systems with elliptic holes (10 nm and $20 \mathrm{~nm}$ cells, respectively). Atoms on opposite sides of the crack faces (in red) ignore themselves.

vicinity of the ground state of the system. Indeed, the reactive ability of REBO is needed only where atomic rearrangements occur, e.g., at a crack tip; elsewhere, the reactive ability is unnecessary and could be replaced by a simpler non-reactive analogue, the computational cost of which could be much less than that of the reactive potential. During a MD, the REBO potential is substituted by the reduced potential on the fly on a bond-per-bond basis in the part of the atomic system where the harmonic approximation is reasonably valid. A seamless coupling is set up at the interface between the two potentials, thus ensuring that a system modeled with the coupled potentials behaves like a system modeled with the reactive potential only. All the details about this methodology are available in (Tejada et al., 2016).

All the molecular simulations were performed with a Langevin thermostat that ensures isothermal conditions at a prescribed temperature of $300 \mathrm{~K}$. Failure is a process that releases a lot of heat and a constant temperature is not a relevant assumption after the onset of failure. Here, isothermal condition is valid because we are interested in the onset of failure only and we disregard the subsequent propagation. Regarding the mechanical loading, a constant strain rate of $0.5 \% / \mathrm{ps}$ was imposed in the vertical direction $(y)$ during the simulation, which is small enough to ensure no rate dependence of the elastic behavior of the 2D model material. Most importantly, we considered 
always the same temperature and the same strain rate for all the molecular simulations. Indeed, failure is a thermally activated process and one expects the stress at failure to decrease with both temperature and loading time (inverse of the strain rate). For instance, Yazdani and Hatami (2015) report the change in the yield surface of graphene in function of temperature. By considering always the same temperature and strain rate, we made sure that those parameters introduce no bias in our analysis. We computed the stress following the usual virial estimate and, in all the results hereafter, we report the values of maximum tensile stress $\Sigma_{c r}$ in the vertical direction $(y)$ at the onset of failure. All the simulations were performed with an in-house code developed previously by Tejada et al. (2016).

We display in Figures 6 the results of failure simulations. Following the dimensionless formalism introduced before, we display the reduced failure stress $\Sigma_{c r} \sqrt{L / 2} / K_{I c}$ in function of the reduced flaw size $2 a / L$. The value of the toughness $K_{I c}=2.5 \cdot 10^{-3}(\mathrm{~N} / \mathrm{m}) \cdot \sqrt{\mathrm{m}}$ was fitted so that the results of pre-cracked systems follow LEFM predictions in the regimes were fracture failure is smaller than yield failure. The same value of toughness was used for both the $10 \mathrm{~nm}$ systems and the $20 \mathrm{~nm}$ systems. The yield limit case is higher for the $20 \mathrm{~nm}$ systems than for the $10 \mathrm{~nm}$ systems because of the size-dependence of yield failure in the dimensionless formulation (Equation $5)$. The characteristic length $l_{0}=\left(K_{\text {Ic }} / \sigma_{\text {yield }}\right)^{2}=1.5 \mathrm{~nm}$ is derived from the value of toughness obtained above and from the value of yield strength $\sigma_{\text {yield }}=65 \mathrm{~N} / \mathrm{m}$ of a flawless strip.

These results deserve some careful analysis. First, one can observe that the results for pre-cracked systems follow fracture failure very well in the regime where yield failure exceeds fracture failure. Interestingly, outside this regime, one can identify two opposite changes: 1- at small crack lengths, deviation from fracture failure is observed and yield failure appears as an upper boundary to the failure stress; 2- at large crack lengths, no deviation is observed, in other words, the failure stress seems to follow fracture failure and exceeds the yield limit of the material. These two observations are more pronounced in the results of the $10 \mathrm{~nm}$ system, for which the reduced cell size $L /\left(2 l_{0}\right)=3.3$ is small enough for the regimes where fracture exceeds yield to be of significant extent. In contrast, for the $20 \mathrm{~nm}$ system $\left(L /\left(2 l_{0}\right)=6.6\right)$, only two of the molecular simulation results enter those regimes. As for systems with initial elliptic holes, we observe a continuous evolution from the fracture failure limit at low aspect ratios $b / a$ to the yield failure limit at large aspect ratios. This time, the evolution is more accurately followed 

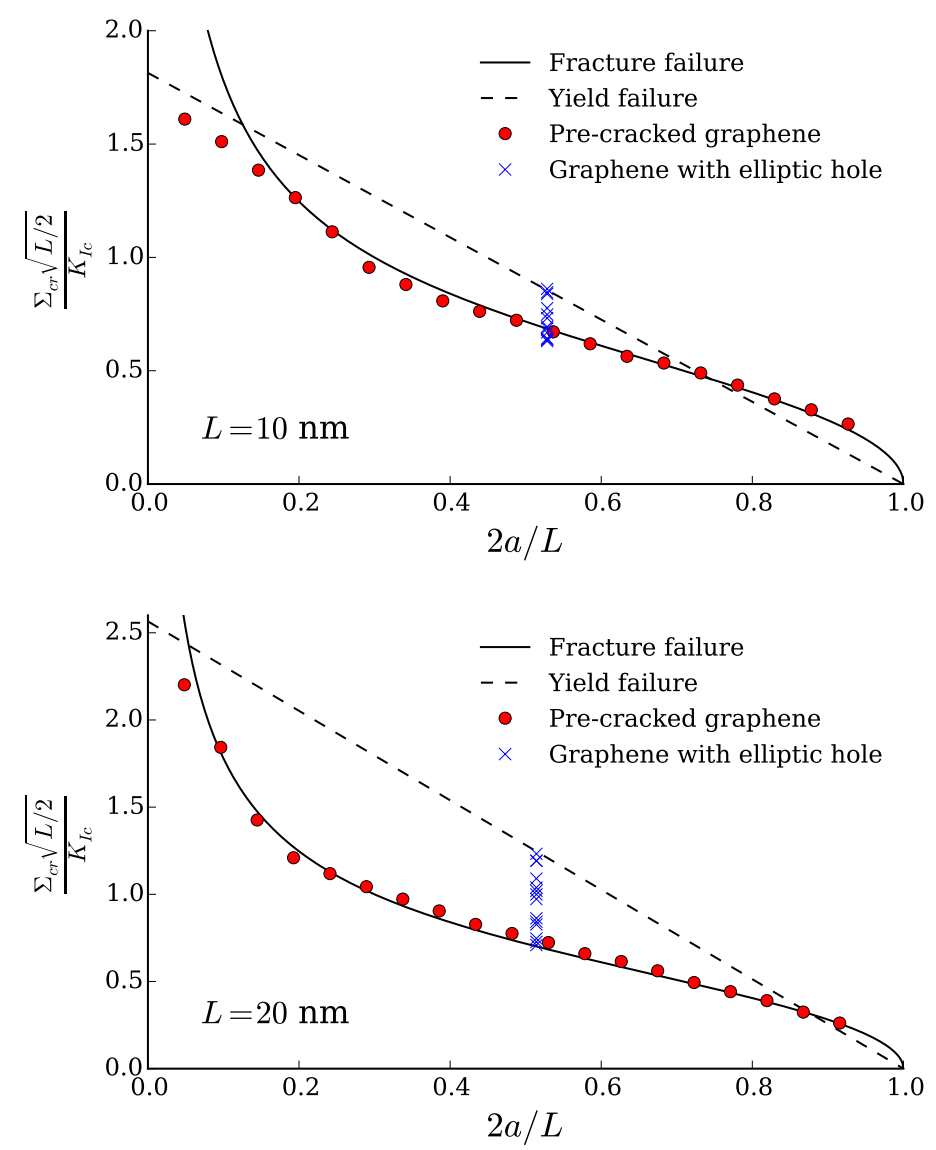

Figure 6: Results of molecular simulations of failure of the $10 \mathrm{~nm}$ (top) and $20 \mathrm{~nm}$ (bottom) systems with initial cracks and elliptic holes of various dimensions, and comparison to the theoretical limit cases of fracture failure and yield failure. 


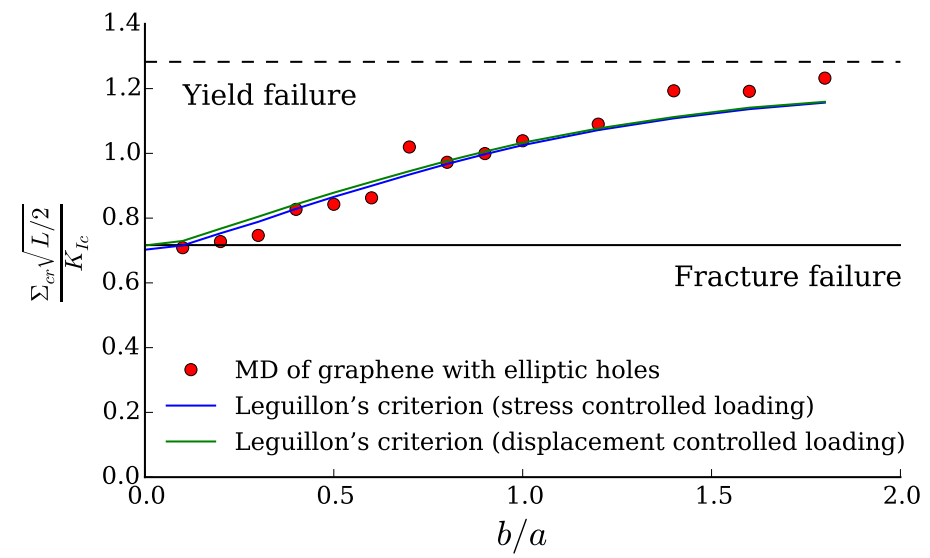

Figure 7: Failure of the $20 \mathrm{~nm}$ systems with elliptic holes, in function of the aspect ratio. Guides are included that represent the limit cases of fracture failure and yield failure. Molecular simulation results are compared to the prediction of the FFM criterion of Leguillon.

for the $20 \mathrm{~nm}$ systems than for the $10 \mathrm{~nm}$ systems, because the larger the system size is, the larger the difference between fracture and yield failures is. For the $20 \mathrm{~nm}$ systems, we display in Figure 7 the critical stress at failure in function of the aspect ratio of the ellipse along with the two limit cases (fracture and yield).

\section{Mechanical analysis and discussion}

Let us discuss the implications of these results on our understanding of failure initiation. Most initiation theories are consistent with the deviation from fracture failure for the failure of pre-cracked systems with small initial crack lengths. However, the observation that failure of pre-cracked systems exceeds yield failure at large crack lengths is remarkable, since many initiation theories consider yield failure as an upper boundary of the stress. One may wonder whether this observation is not an artifact because, in such situations, periodic crack tips are very close from one another which could trigger unexpected behaviors such as the overlap of surface effects. Nevertheless, failure starts exceeding yield when periodic crack tips are separated by a distance of $2 \mathrm{~nm}$, i.e., about 10 atoms. Surface effects are usually limited to the very first layers of atoms, which make it unlikely to observe overlap at a distance of $2 \mathrm{~nm}$ (see Figure 11 discussed later). Although, overlap may ex- 
ist for shorter distances $(\sim 1 \mathrm{~nm})$, the observation that failure exceeds yield does not seem to arise from an artifact. This is the case of CZM and damage approaches. Non-local approaches allow the stress to exceeds the yield strength locally, but not on average, whereas, in the above case, the average stress along the horizontal axis $(\mathrm{x})$ between periodic crack tips exceeds the yield failure. As for FFM approaches, some of the proposed criteria allow for failure at stresses higher than yield, in particular the criterion of Leguillon (2002). An essential consideration at the origin of this criterion is that strength and critical energy released are both necessary conditions of failure. According to this postulate, the stress in a system can exceed the yield strength if the energy released by the failure is less than the critical energy release rate. Leguillon (2002) gives the example of the experiments of Parvizi et al. (1978) on constrained failure of composites of various thicknesses: it shows that, at small thicknesses, failure occurs at strains that exceeds the yield limit and follow an energy released criterion while, at large thicknesses, yield failure is observed. In the FFM approach of Li et al. (1995), the authors propose a modification of the stress part of the criterion to allow for stresses higher than the yield strength when the energy released part of the criterion cannot be fulfilled. This modification comes with an additional parameter (multiplicative constant to apply to the strength in the stress criterion). But how this new strength is related to the material and structure properties remains unspecified, which does not offer a systematic failure criterion, in contrast with the criterion of Leguillon (2002).

In the light of Leguillon's approach, one can qualitatively understand why failure exceeds yield at large crack lengths: pre-cracked systems with large initial cracks strongly concentrate the stress in-between the periodic cracks while storing little mechanical energy because of their compliance. Therefore, in such systems, a criterion on stress is more easily reached than a criterion on energy. Energy is the limiting factor in failure initiation, and stress must exceed strength before enough energy can be released by failure.

Following FFM and Leguillon's criterion, let us consider the initiation of a crack of length $l$ from the existing flaw (see the drawings in Figure 8). Here, for simplicity, we assume symmetry of the initiation, whereas it is likely that initiation would start first on one side of the initial flow only. Failure of the periodic cell is highly unstable and cracking immediately connects the periodic flaws, irrespective of the symmetry of initiation, which makes it impossible to analyze from the molecular simulation results. Assuming symmetric initiation is expected to have little effect on the results at small aspect 
ratios $b / a$. But, the results at large aspect ratios may need confirmation by considering asymmetric initiation. This is left for further developments.

Assuming linear elasticity, the macroscopic behavior of the periodic system is of the form:

$$
\Sigma=E D\left(\frac{2 l}{L}\right) \bar{\varepsilon}
$$

where $E$ is the Young's modulus, $\bar{\varepsilon}$ is the average (macroscopic) tensile strain in the vertical direction $(y)$, and $D$ is a dimensionless factor accounting for the presence of the periodic flaw which depends on the geometric ratios $2 a / L$ and $b / a$ (in the limit $2 a / L \rightarrow 0$ of a flawless body, $D=1 /\left(1-\nu^{2}\right)$, with $\nu$ the Poisson's ratio). We display in Figure 8 the values of the factor $D$ for the systems studied here. The values for systems with initial ellipse were computed by FEM calculations with the Code_Aster software package (http://web-code-aster.org). And the values for systems with initial crack (and infinitely thin ellipse $b / a=0$ ) were computed with the pseudo-traction method of Karihaloo et al. (1996). We display here the results for a Poisson's ratio of $\nu=0.3$.

Owing to Hill's lemma in the case of periodic heterogeneous materials (Michel et al., 1999), the complementary energy of the periodic cell is:

$$
P=-\frac{1}{2} \frac{L^{2} \Sigma^{2}}{E \cdot D(2 l / L)}
$$

The energy released by initiation at constant loading $\Sigma$ is:

$$
\Delta P=P(0)-P(2 l / L)=\frac{1}{2} \frac{L^{2} \Sigma^{2}}{E}\left(-\frac{1}{D(0)}+\frac{1}{D(2 l / L)}\right)
$$

Following Finite Fracture Mechanics, failure can occur if this energy exceeds the critical energy release rate $2 l G_{c}$ (the factor 2 comes from the fact two cracks initiate symmetrically on both sides of the initial flaw). This leads to a first condition for initiation:

$$
f\left(\frac{2 l}{L}\right) \geq \frac{2 l_{0}}{L}\left(\frac{\sigma_{\text {yield }}}{\Sigma}\right)^{2} \text { with } f(u)=\frac{1}{u}\left(-\frac{1}{D(0)}+\frac{1}{D(u)}\right)
$$

where we introduced the length $l_{0}=E G_{c} / \sigma_{\text {yield }}^{2}=\left(K_{I c} / \sigma_{\text {yield }}\right)^{2}$ characterizing the size of the yielding zone at a crack tip. Leguillon (2002) introduces a second condition on stress following the intuition that strength and toughness 

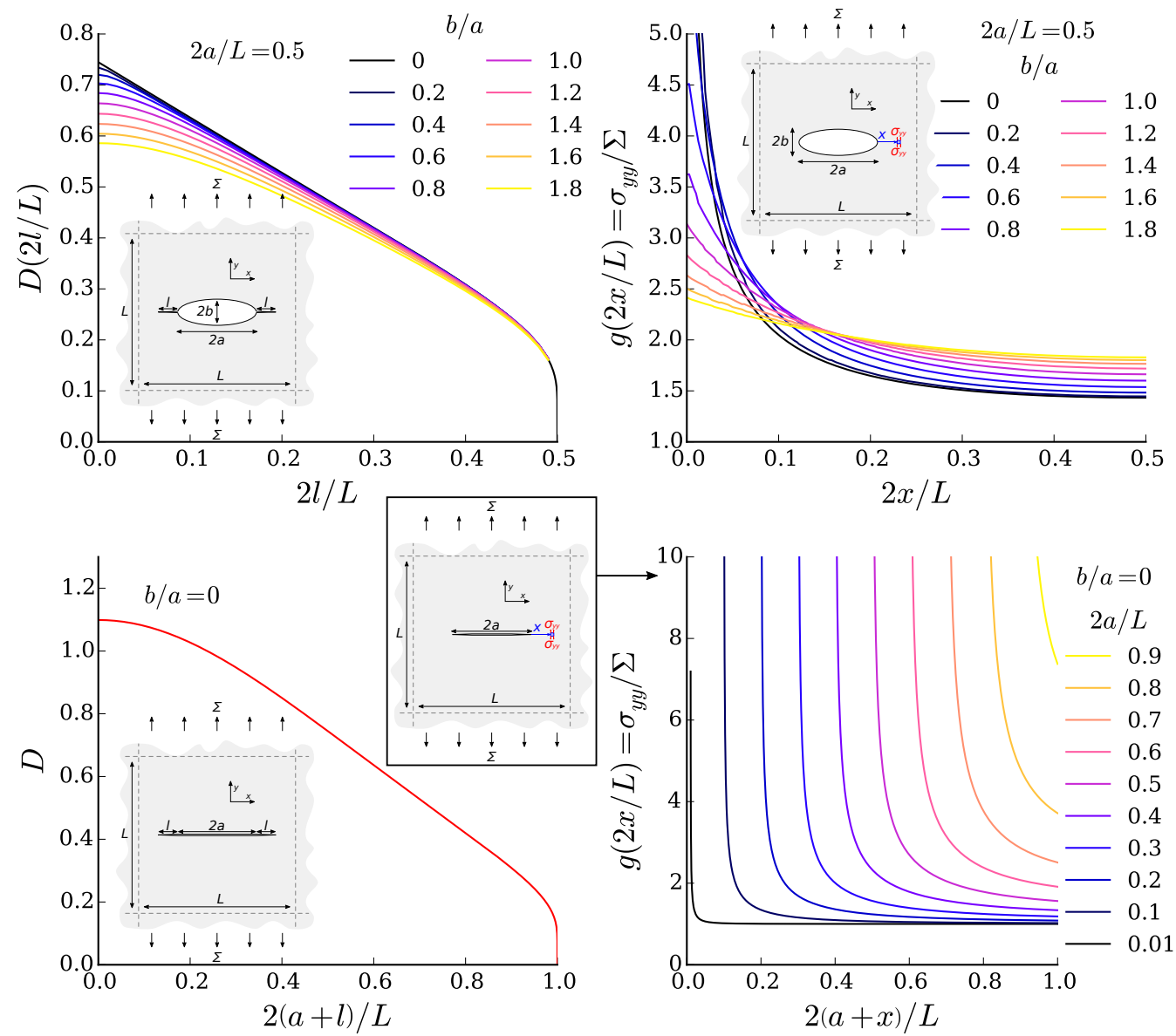

Figure 8: Reduced elasticity $D$ for the systems with initial ellipse (upper left) and initial crack (lower left); and stress profile $g$ along the path of crack initiation for the systems with initial ellipse (upper right) and initial crack (lower right). 
are both necessary conditions of failure. The second condition proposed by Leguillon (2002) is that stress prior to failure must exceed the yield strength over the path of crack initiation:

$$
\sigma_{y y}(x, y=0) \geq \sigma_{y i e l d}, \forall x \in \text { path of crack initiation }
$$

With the symmetry of the problem, the stress is of the form: $\sigma_{y y}=$ $g(2 x / L) \cdot \Sigma$, where $g$ is a dimensionless function which depends on the geometric ratios $2 a / L$ and $b / a$ and which decreases with $2 x / L$. We display in Figure 8 the value of the function $g$ computed with the numerical approaches as for $D$. The second criterion on stress thus takes the form:

$$
g\left(\frac{2 l}{L}\right) \geq \frac{\sigma_{\text {yield }}}{\Sigma}
$$

For a given loading Sigma, the first condition (Equation 9) provides a lower boundary of the length of initiation since the function $f$ is increasing with $2 l / L$ (more precisely, $f=O\left((2 l / L)^{\alpha}\right)$ with $0<\alpha<1, \alpha=1$ being the limit of initiation from a flat surface and $\alpha=0$ the limit of initiation from a crack, and any moderate stress concentration is in-between these two limits). Conversely, the second condition (Equation 11) provides an upper boundary of the length of initiation $l$ since the function $g$ is decreasing with $2 l / L$. We have:

$$
\frac{2 l}{L} \geq f^{-1}\left(\frac{2 l_{0}}{L}\left(\frac{\sigma_{\text {yield }}}{\Sigma}\right)^{2}\right) \text { and } \frac{2 l}{L} \leq g^{-1}\left(\frac{\sigma_{\text {yield }}}{\Sigma}\right)
$$

Failure occurs when the loading $\Sigma$ is strong enough for both conditions to be compatible (see Figure 9 ). The critical loading $\Sigma_{c r}$ verifies:

$$
f^{-1}\left(\frac{2 l_{0}}{L}\left(\frac{\sigma_{\text {yield }}}{\Sigma_{c r}}\right)^{2}\right)=g^{-1}\left(\frac{\sigma_{\text {yield }}}{\Sigma_{c r}}\right)
$$

Solving for equation 13 provides the critical loading at failure. Alternatively, one could solve for the critical initiation length $2 l_{c r} / L$ instead, which verifies:

$$
f\left(\frac{2 l_{c r}}{L}\right)=\frac{2 l_{0}}{L}\left(g\left(\frac{2 l_{c r}}{L}\right)\right)^{2}
$$




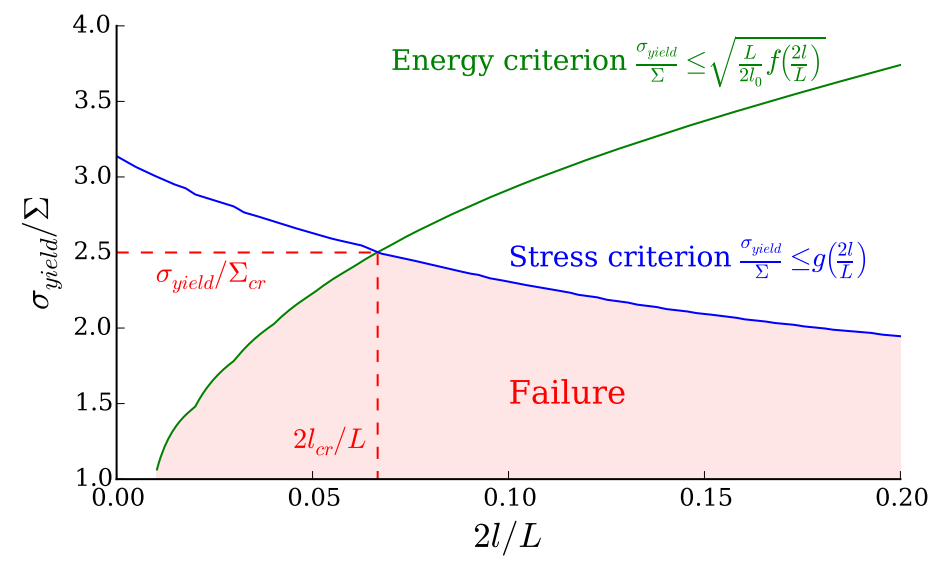

Figure 9: Representation of the combined stress-energy criterion in the case of failure initiation from a circular hole $(b / a=1)$. The colored area corresponds to the cases where both energy and stress criteria are satisfied. Failure stress is obtained at the intersection of the two criteria.

Note that, in Finite Fracture Mechanics, the failure criterion depends on the nature of loading (force or displacement). In usual Lineal Elastic Fracture Mechanics, this is not the case. Considering a loading in displacement of the periodic cell (i.e., a macroscopic strain is imposed), the compatibility Equations 13 and 14 verified by the critical loading and initial length are modified as follows:

$$
f^{*-1}\left(\frac{2 l_{0}}{L}\left(\frac{\sigma_{y i e l d}}{\Sigma_{c r}}\right)^{2}\right)=g^{-1}\left(\frac{\sigma_{\text {yield }}}{\Sigma_{c r}}\right) \text { and } f^{*}\left(\frac{2 l_{c r}}{L}\right)=\frac{2 l_{0}}{L}\left(g\left(\frac{2 l_{c r}}{L}\right)\right)^{2}
$$

where $f^{*}(u)=(1 / u) \cdot(D(0)-D(u)) /(D(0))^{2}$.

We compare in Figure 7 the predictions of Leguillon's combined criterion to the molecular simulation results of failure initiation from the elliptic holes. Both loading conditions (stress controlled and displacement controlled) are displayed. Since the criterion's only parameters are the toughness and yield stress, no fitting was performed. The criterion captures failure initiation reasonably well. We observe some over-estimation at small aspect ratios and under-estimation at large aspect ratios, but these are moderate with respect to the inherent variability of the molecular simulation results.

A particular situation of interest, is the limit case of an initial crack 
$(b / a=0)$. In that case, the condition on stress (Equation 11) imposes a minimum initiation length: $g(2 x / L) \approx \sqrt{\frac{a C(2 a / L)}{2 x}} \Rightarrow \frac{l}{a} \geq \frac{1}{2} C\left(\frac{2 a}{L}\right)\left(\frac{\Sigma}{\sigma_{\text {yield }}}\right)^{2}$. Accordingly, the initiation length is very small as long as failure occurs at stresses much smaller than the yield $\left(\Sigma / \sigma_{\text {yield }}\right.$ small). Assuming this is the case, the energy released by an infinitesimal initiation is the usual energy release rate of fracture mechanics: $\Delta P \approx 2 l G$. Thus, the condition on energy released (Equation 9 ) becomes $G \geq G_{c}$, which is the usual criterion of fracture mechanics. Accordingly, this initiation criterion is consistent with fracture mechanics. Deviation from fracture mechanics arises when the crack length is very small or very large compared to the cell size $(2 a / L \rightarrow 0$ and $2 a / L \rightarrow$ 1). For small crack lengths, the initiation length is no more infinitesimal and therefore the energy criterion is no more equivalent to usual fracture mechanics. In the limit $2 a / L=0$, one recovers the yield failure. For large crack lengths, the maximum initiation length of the criterion on stress can exceed the length of intact material: $g^{-1}\left(\sigma_{\text {yield }} / \Sigma\right) \geq 1-2 a / L$. In this situation, all the mechanical energy available in the system is not enough to satisfy the energy criterion upon complete failure while the stress criterion is satisfied all along the intact material. The initiation length equals the length of intact material $2 l / L=1-2 a / L$ and only the criterion on energy has to be satisfied:

$$
f^{-1}\left(\frac{2 l_{0}}{L}\left(\frac{\sigma_{y i e l d}}{\Sigma_{c r}}\right)^{2}\right)=1-\frac{2 a}{L}
$$

where the function $f$ must be replaced by the function $f^{*}$ for loading in displacement. We compare in Figure 10 the predictions of Leguillon's criterion (displacement loading) to the results of molecular simulation of failure for the $10 \mathrm{~nm}$ systems with initial cracks. Transition from fracture failure to yield failure at small and large crack lengths is qualitatively consistent with MD results. In particular, failure exceeds yield at large crack lengths and not at small crack lengths. As discussed before, predicting a failure that exceeds yield failure is uncommon among initiation criteria and is quite specific to the approach of Leguillon (2002). Quantitatively, the criterion tends to underestimate failure at small and large crack lengths where failure deviates from fracture failure. This comparison along with the previous one (Figure 7) tend to support the approach proposed by Leguillon (2002). The idea of a combined stress-energy criterion captures the main feature of failure initiation in this example of a 2D model material. Of course, the precise 


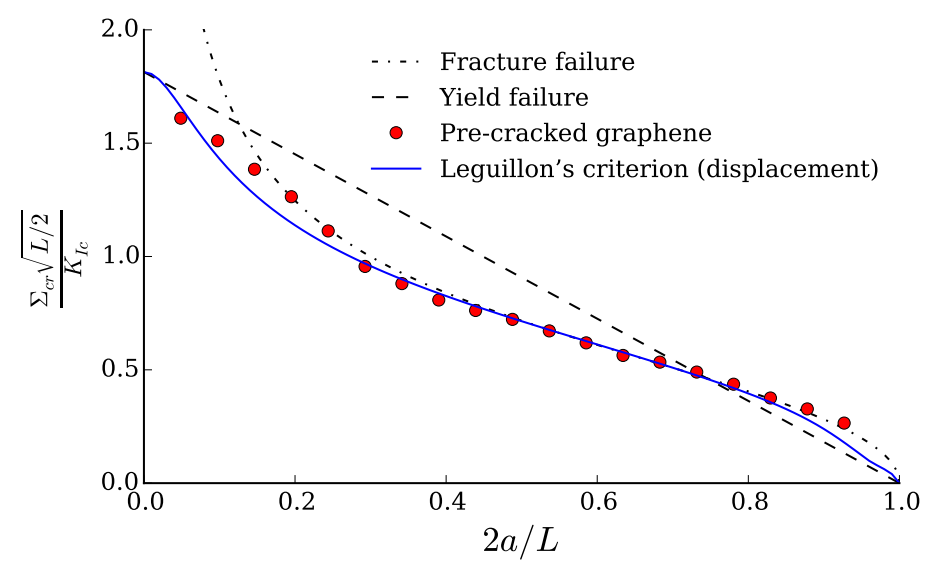

Figure 10: Comparison of the FFM criterion of Leguillon with the results of molecular simulation of failure of the $10 \mathrm{~nm}$ systems with initial cracks, in function of the crack length. For the comparison, we also recall the limit cases of fracture failure and yield failure.

formulation could be improved to reach a better quantitative comparison, but the general principle describes well these molecular simulation results.

Interestingly, previous molecular studies of initiation (Mattoni et al., 2005; Lu et al., 2008; Zhang et al., 2012; Brochard et al., 2015; Yin et al., 2015) all observed failure that cannot exceed yield. Actually, none of these works has focused on such systems with low mechanical energy and high stress concentration. Although, Leguillon's criterion offers an interesting interpretation of this phenomenon, other equally valid interpretations might be possible, for instance based on surface effects. We display in Figure 11 the distribution of stress on a per atom basis just before failure in two of the simulated $10 \mathrm{~nm}$ systems: a system with large crack, for which yield limit is exceeded, and a system with an elliptic hole with high aspect ratio, which approaches the yield limit. One observes that the most stressed atoms are surface atoms. For the system with an elliptic hole, one could expect that stress would distribute almost evenly in the intact region, but this is not the case and surface atoms are clearly more solicited. Therefore one can reasonably think that surface effects are key to the initiation behavior. Note that the yield strength we considered is that of a strip, i.e., non periodic in the direction orthogonal to loading. Accordingly, surface effects also contribute to the strength of the material. However, for a more in depth understanding of the role of surface effect on failure, further investigation is needed. 

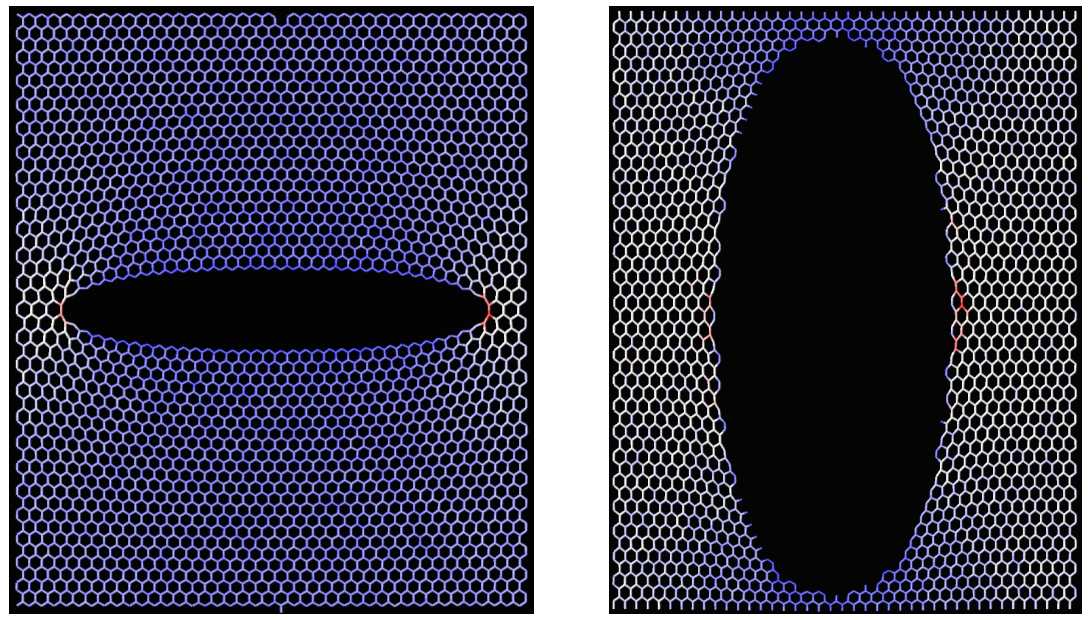

Figure 11: Snapshots of the distribution of volumetric stress just before failure for a system with large crack, for which yield limit is exceeded, (left), and for a system with an elliptic hole with high aspect ratio, which approaches the yield limit (right).

\section{Conclusion}

In this work, we investigate the process of failure initiation by molecular simulation considering the particular case of a 2D model material. We show that the transition from fracture failure to yield failure can be captured if the system size $L$, usually limited to a few tenth of nanometer for molecular simulation studies, is significantly larger than the characteristic length $l_{0}=\left(K_{I c} / \sigma_{\text {yield }}\right)^{2}$, which characterizes the size of the process zone at a crack tip. For large values of $L /\left(2 l_{0}\right)$ (e.g., 6.6 for the $20 \mathrm{~nm}$ systems) one can well capture failure initiation from non crack-like flaws (elliptic holes in the present case), but the transition from fracture to yield is difficult to characterize when considering failure from very small $(2 a / L \rightarrow 0)$ or very large $(2 a / L \rightarrow 1)$ initial cracks. Conversely, for moderate values of $L /\left(2 l_{0}\right)$ (e.g., 3.3 for the $10 \mathrm{~nm}$ systems), transition from fracture to yield is less pronounced when considering initiation from an elliptic flaw, but more pronounced when considering the transition at small and large initial cracks. Confronting our results of molecular simulations with existing theories, we identify a peculiarity of the initiation behavior that most initiation theories would not predict: the failure exceeds the yield failure in some cases where the initial flaw strongly concentrates stresses while little mechanical energy is stored in the material (initiation from large initial cracks). We show that 
the failure behavior we observe is consistent with one specific Finite Fracture Mechanics initiation theory, that of Leguillon (2002), the formulation of which authorizes failure that exceeds yield failure in these peculiar cases. The key principle at the heart of this theory is that failure occurs when both a stress criterion (strengh) and an energy criterion (toughness) are satisfied for the initiation of a crack over a finite length. Our results of molecular simulations are consistent with this interpretation. Of course, this work is focused on a particular model material, under a peculiar loading, stretching in one direction with restrained deformation in the other direction. Further investigation is needed to confirm these results and deepen our understanding of failure initiation. Various phenomena at the atomic scale could explain why the stress can locally exceed yield, from purely statistical reasons (because of temperature, atoms are not in static equilibrium) to chemistry (the atomic environment near a flaw differs from that in the bulk giving rise to unusual behaviors such as surface effects) and lattice geometry (the atomic scale is not a continuum and failure is governed by bonds breaking between discrete atoms on a peculiar lattice geometry). In particular, the stress distribution at the onset of failure suggests that surface effects are likely to play a key role in the model system studied. How surface effects impact the failure behavior, and how this articulates with Leguillon's criterion based on energy and stress, are open questions that require further investigation.

\section{Acknowledgment}

We gratefully acknowledge funding from the Labex MMCD provided through the national program Investments for the Future of the French National Research Agency (ANR-11-LABX-022-01)

Anderson, T. L., 2005. Fracture Mechanics: Fundamentals and Applications. CRC Press, Taylor \& Francis Group.

Barenblatt, G. I., 1962. The mathematical theory of equilibrium cracks in brittle fracture. Advances in applied mechanics 7 (1), 55-129.

Bažant, Z. P., 1984. Size Effect in Blunt Fracture: Concrete, Rock, Metal. Journal of Engineering Mechanics 110 (4), 518-535.

Bažant, Z. P., Cedolin, L., 1979. Blunt crack band propagation in finite element analysis. Journal of the Engineering Mechanics Division 105 (2), $297-315$. 
Bažant, Z. P., Cedolin, L., 1980. Fracture mechanics of reinforced concrete. Journal of the Engineering Mechanics Division 106 (6), 1287-1306.

Bažant, Z. P., Cedolin, L., jan 1983. Finite Element Modeling of Crack Band Propagation. Journal of Structural Engineering 109 (1), 69-92.

URL http://ascelibrary.org/doi/10.1061/(ASCE) 0733-9445(1983) 109:1 (69)

Belytschko, T., Xiao, S. P., Schatz, G. C., Ruoff, R. S., 2002. Atomistic simulations of nanotube fracture. Physical Review B 65 (23), 235430.

URL http://link.aps.org/doi/10.1103/PhysRevB.65.235430

Bilby, B. A., Cottrell, A. H., Swinden, K. H., 1963. The Spread of Plastic Yield from a Notch. Proceedings of the Royal Society of London. Series A. Mathematical and Physical Sciences 272 (1350), 304-314.

URL http://rspa.royalsocietypublishing.org/content/272/1350/304.abstract

Bourdin, B., Francfort, G. A., Marigo, J.-J., apr 2008. The Variational Approach to Fracture. Journal of Elasticity 91 (1-3), 5-148.

URL http://link.springer.com/10.1007/s10659-007-9107-3

Brenner, D. W., Shenderova, O. A., Harrison, J. A., Stuart, S. J., Ni, B., Sinnott, S. B., feb 2002. A second-generation reactive empirical bond order (REBO) potential energy expression for hydrocarbons. Journal of Physics: Condensed Matter 14 (4), 783-802.

URL http://stacks. iop.org/0953-8984/14/i=4/a=312?key=crossref . 563ba867ec801ce55

Brochard, L., Hantal, G., Laubie, H., Ulm, F.-J., Pellenq, R. J. M., aug 2015. Capturing material toughness by molecular simulation: accounting for large yielding effects and limits. International Journal of Fracture 194 (2), 149-167.

URL http://link.springer.com/10.1007/s10704-015-0045-y

Cao, A., Wei, Y., jul 2007. Atomistic simulations of crack nucleation and intergranular fracture in bulk nanocrystalline nickel. Physical Review B 76 (2), 024113.

URL http: //www . scopus. com/inward/record. url?eid=2-s2.0-34547105560\&partnerID=tZ http://link.aps.org/doi/10.1103/PhysRevB.76.024113

Cedolin, L., Bažant, Z. P., 1980. Effect of finite element choice in blunt crack band analysis. Computer Methods in Applied Mechanics and Engineering 24, 305-316. 
Chaboche, J.-L., 1981. Continuous damage mechanics A tool to describe phenomena before crack initiation. Nuclear Engineering and Design 64 (2), 233-247.

Charlotte, M., Laverne, J., Marigo, J.-J., 2006. Initiation of cracks with cohesive force models: a variational approach. European Journal of Mechanics, A/Solids 25 (4), 649-669.

Dewapriya, M. A. N., Rajapakse, R. K. N. D., Phani, A. S., jun 2014. Atomistic and continuum modelling of temperature-dependent fracture of graphene. International Journal of Fracture 187 (2), 199-212.

URL http://link. springer.com/10.1007/s10704-014-9931-y

Dugdale, D., 1960. Yielding of steel sheets containing slits. Journal of the Mechanics and Physics of Solids 8 (2), 100-104.

Ferdjani, H., Abdelmoula, R., Marigo, J. J., aug 2007. Insensitivity to small defects of the rupture of materials governed by the Dugdale model. Continuum Mechanics and Thermodynamics 19 (3-4), 191-210.

URL http://link. springer.com/10.1007/s00161-007-0051-z

Francfort, G., Marigo, J.-J., aug 1998. Revisiting brittle fracture as an energy minimization problem. Journal of the Mechanics and Physics of Solids 46 (8), 1319-1342.

URL http://linkinghub.elsevier.com/retrieve/pii/S0022509698000349

Fyta, M. G., Remediakis, I. N., Kelires, P. C., Papaconstantopoulos, D. a., may 2006. Insights into the Fracture Mechanisms and Strength of Amorphous and Nanocomposite Carbon. Physical Review Letters 96 (18), 185503.

URL http://link.aps.org/doi/10.1103/PhysRevLett.96.185503

Gao, H., 2006. Application of fracture mechanics concepts to hierarchical biomechanics of bone and bone-like materials. International Journal of Fracture 138 (1-4), 101-137.

Gao, H., Chen, S., 2005. Flaw Tolerance in a Thin Strip Under Tension. Journal of Applied Mechanics 72 (5), 732-737. 
Gao, H., Ji, B., Jager, I. L., Arzt, E., Fratzl, P., 2003. Materials become insensitive to flaws at nanoscale: lessons from nature. Proceedings of the National Academy of Sciences 100 (10), 5597-5600.

Griffith, A. A., jan 1921. The Phenomena of Rupture and Flow in Solids. Philosophical Transactions of the Royal Society A: Mathematical, Physical and Engineering Sciences 221 (582-593), 163-198. URL http://rsta.royalsocietypublishing.org/cgi/doi/10.1098/rsta.1921.0006

Gu, X. W., Wu, Z., Zhang, Y.-w., Srolovitz, D. J., Greer, J. R., nov 2013. Microstructure versus Flaw: Mechanisms of Failure and Strength in Nanostructures. Nano Letters 13 (11), 5703-5709.

URL http://pubs.acs.org/doi/abs/10.1021/nl403453h

Hashin, Z., jul 1996. Finite thermoelastic fracture criterion with application to laminate cracking analysis. Journal of the Mechanics and Physics of Solids 44 (7), 1129-1145.

URL http://linkinghub.elsevier.com/retrieve/pii/0022509695000801

Hillerborg, A., Modéer, M., Petersson, P.-E., nov 1976. Analysis of crack formation and crack growth in concrete by means of fracture mechanics and finite elements. Cement and Concrete Research 6 (6), 773-781. URL http://linkinghub. elsevier .com/retrieve/pii/0008884676900077

Inglis, C. E., 1913. Stresses in a plate due to the presence of cracks and sharp corners. Transactions of the Institute of Naval Architects 55, 219-241.

Inoue, H., Akahoshi, Y., Harada, S., 1995. Molecular dynamics simulation on fracture mechanisms of nano-scale polycrystal under static and cyclic loading. Journal of the Society of Materials Science, Japan 44 (501Appendix), 95-99. URL http://joi.jlc.jst.go.jp/JST. Journalarchive/jsms1963/44.501Appendix_95?from=

Irwin, G. R., 1957. Analysis of Stresses and Strains Near the End of a Crack Traversing a Plate. Journal of Applied Mechanics 24, 361-364.

Karihaloo, B. L., Wang, J., Grzybowski, M., oct 1996. Doubly periodic arrays of bridged cracks and short fibre-reinforced cementitious composites. Journal of the Mechanics and Physics of Solids 44 (10), 1565-1586. URL http://linkinghub.elsevier.com/retrieve/pii/0022509696000531 
Kumar, S., Li, X., Haque, A., Gao, H., 2011. Is Stress Concentration Relevant for Nanocrystalline Metals? Nano Letters 11 (6), 2510-2516.

URL http://pubs.acs.org/doi/abs/10.1021/nl201083t

Laverne, J., Marigo, J.-J., apr 2004. Approche globale, minima relatifs et Critère d'Amorçage en Mécanique de la Rupture. Comptes Rendus Mécanique 332 (4), 313-318.

URL http://linkinghub.elsevier .com/retrieve/pii/S1631072104000427

Leguillon, D., 2002. Strength or toughness? A criterion for crack onset at a notch. European Journal of Mechanics, A/Solids 21 (1), 61-72.

Leguillon, D., Piat, R., may 2008. Fracture of porous materials Influence of the pore size. Engineering Fracture Mechanics 75 (7), 1840-1853.

URL http: //linkinghub.elsevier.com/retrieve/pii/S0013794406004541

Leguillon, D., Quesada, D., Putot, C., Martin, E., oct 2007. Prediction of crack initiation at blunt notches and cavities size effects. Engineering Fracture Mechanics 74 (15), 2420-2436.

URL http://linkinghub.elsevier .com/retrieve/pii/S0013794406004310

Lemaitre, J., 1986. Local approach of fracture. Engineering Fracture Mechanics 25 (5-6), 523-537.

Li, J., Zhang, X., mar 2006. A criterion study for non-singular stress concentrations in brittle or quasi-brittle materials. Engineering Fracture Mechanics 73 (4), 505-523.

URL http://linkinghub.elsevier.com/retrieve/pii/S0013794405002249

Li, Y. N., Hong, A. P., Bažant, Z. P., dec 1995. Initiation of parallel cracks from surface of elastic half-plane. International Journal of Fracture 69 (4), 357-369.

URL http://link. springer.com/10.1007/BF00037384

Lu, Q., Marks, N., Schatz, G. C., Belytschko, T., jan 2008. Nanoscale fracture of tetrahedral amorphous carbon by molecular dynamics: Flaw size insensitivity. Physical Review B 77 (1), 014109.

URL http://link.aps.org/doi/10.1103/PhysRevB.77.014109

Marigo, J.-J., dec 2010. Initiation of Cracks in Griffiths Theory: An Argument of Continuity in Favor of Global Minimization. Journal of Nonlinear 
Science 20 (6), 831-868.

URL http://link.springer.com/10.1007/s00332-010-9074-x

Marigo, J.-J., Truskinovsky, L., may 2004. Initiation and propagation of fracture in the models of Griffith and Barenblatt. Continuum Mechanics and Thermodynamics 16 (4), 391-409.

URL http://link.springer.com/10.1007/s00161-003-0164-y

Martin, E., Leguillon, D., Carrère, N., dec 2012. A coupled strength and toughness criterion for the prediction of the open hole tensile strength of a composite plate. International Journal of Solids and Structures 49 (26), 3915-3922.

URL http://www.sciencedirect.com/science/article/pii/S0020768312003654 http://linkinghub.elsevier.com/retrieve/pii/S0020768312003654

Mattoni, a., Colombo, L., Cleri, F., 2005. Atomic scale origin of crack resistance in brittle fracture. Physical Review Letters 95 (11), 1-4.

Maugis, D., sep 1992. Stresses and displacements around cracks and elliptical cavities: Exact solutions. Engineering Fracture Mechanics 43 (2), 217-255. URL http://linkinghub.elsevier.com/retrieve/pii/001379449290123V

Michel, J., Moulinec, H., Suquet, P., apr 1999. Effective properties of composite materials with periodic microstructure: a computational approach. Computer Methods in Applied Mechanics and Engineering 172 (1-4), 109-143.

URL http://linkinghub.elsevier.com/retrieve/pii/S0045782598002278

Moura, M. J. B., Marder, M., 2013. Tearing of free-standing graphene. Physical Review E 88 (3), 032405.

URL http://link.aps.org/doi/10.1103/PhysRevE.88.032405

Novozhilov, V., jan 1969. On a necessary and sufficient criterion for brittle strength. Journal of Applied Mathematics and Mechanics 33 (2), 201-210. URL http://linkinghub.elsevier.com/retrieve/pii/0021892869900252

Pan, Z., Rupert, T. J., oct 2014. Damage nucleation from repeated dislocation absorption at a grain boundary. Computational Materials Science 93, 206-209.

URL http://www.sciencedirect.com/science/article/pii/S0927025614004790 \n/home/g http://linkinghub.elsevier.com/retrieve/pii/S09270256140047 
Parvizi, A., Garrett, K. W., Bailey, J. E., jan 1978. Constrained cracking in glass fibre-reinforced epoxy cross-ply laminates. Journal of Materials Science 13 (1), 195-201.

URL http://linkinghub.elsevier.com/retrieve/pii/001043617890215X http://link.springer.com/10.1007/BF00739291

Ritchie, R. O., Buehler, M. J., Hansma, P., 2009. Plasticity and toughness in bone. Physics Today 62 (6), 41-47.

Ritchie, R. O., Knott, J. F., Rice, J. R., 1973. On the relationship between critical tensile stress and fracture toughness in mild steel. Journal of the Mechanics and Physics of Solids 21, 395-410.

Romani, R., Bornert, M., Leguillon, D., Le Roy, R., Sab, K., may 2015. Detection of crack onset in double cleavage drilled specimens of plaster under compression by digital image correlation Theoretical predictions based on a coupled criterion. European Journal of Mechanics - A/Solids 51, 172-182. URL http://linkinghub .elsevier.com/retrieve/pii/S0997753814001831

Seweryn, A., mar 1994. Brittle fracture criterion for structures with sharp notches. Engineering Fracture Mechanics 47 (5), 673-681.

URL http://linkinghub.elsevier.com/retrieve/pii/0013794494901589

Seweryn, A., apr 1998. A non-local stress and strain energy release rate mixed mode fracture initiation and propagation criteria. Engineering Fracture Mechanics 59 (6), 737-760.

URL http://linkinghub.elsevier.com/retrieve/pii/S0013794497001756

Seweryn, A., Mróz, Z., aug 1995. A non-local stress failure condition for structural elements under multiaxial loading. Engineering Fracture Mechanics 51 (6), 955-973.

URL http://www.sciencedirect.com/science/article/pii/001379449400335F http://linkinghub.elsevier.com/retrieve/pii/001379449400335F

Shenderova, O. a., Brenner, D. W., Omeltchenko, a., Su, X., Yang, L. H., 2000. Atomistic modeling of the fracture of polycrystalline diamond. Physical Review B 61 (6), 3877-3888.

URL http://link.aps.org/doi/10.1103/PhysRevB.61.3877\nhttp://prb.aps.org/abstra

Tejada, I. G., Brochard, L., Lelièvre, T., Stoltz, G., Legoll, F., Cancès, E., 2016. Coupling a reactive potential with a harmonic approximation for 
atomistic simulations of material failure. Accepted in Computer Methods in Applied Mechanics and Engineering.

Yazdani, H., Hatami, K., sep 2015. Failure criterion for graphene in biaxial loadinga molecular dynamics study. Modelling and Simulation in Materials Science and Engineering 23 (6), 065004.

URL http://stacks. iop.org/0965-0393/23/i=6/a=065004?key=crossref . 66817 f77185033

Yin, H., Qi, H. J., Fan, F., Zhu, T., Wang, B., Wei, Y., 2015. Griffith criterion for brittle fracture in graphene. Nano Letters 15 (3), 1918-1924.

Zhang, T., Li, X., Kadkhodaei, S., Gao, H., 2012. Flaw insensitive fracture in nanocrystalline graphene. Nano Letters 12 (9), 4605-4610.

Zhao, H., Min, K., Aluru, N. R., 2009. Size and chirality dependent elastic properties of graphene nanoribbons under uniaxial tension. Nano Letters 9 (8), 3012-3015. 